\title{
STABILITY OF THE EXIT TIME FOR LÉVY PROCESSES
}

\author{
PHILIP S. GRIFFIN, ${ }^{*}$ Syracuse University \\ ROSS A. MALLER, ${ }^{* *}$ Australian National University
}

\begin{abstract}
This paper is concerned with the behaviour of a Lévy process when it crosses over a positive level, $u$, starting from 0 , both as $u$ becomes large and as $u$ becomes small. Our main focus is on the time, $\tau_{u}$, it takes the process to transit above the level, and in particular, on the stability of this passage time; thus, essentially, whether or not $\tau_{u}$ behaves linearly as $u \downarrow 0$ or $u \rightarrow \infty$. We also consider the conditional stability of $\tau_{u}$ when the process drifts to $-\infty$ almost surely. This provides information relevant to quantities associated with the ruin of an insurance risk process, which we analyse under a Cramér condition.

Keywords: Lévy process; passage time above a level; stability; insurance risk process; Cramér condition; overshoot

2010 Mathematics Subject Classification: Primary 60G51; 60K05

Secondary 91B30
\end{abstract}

\section{Introduction}

For a random walk $S$ starting from 0 with a positive step length distribution and having finite mean, the number of steps required to first pass a positive level $u, \tau_{u}^{S}$, say, is, for large $u$, asymptotic to a multiple of $u$, the constant of proportionality being the reciprocal of the mean step length. More precisely, $\tau_{u}^{S} / u \rightarrow 1 / c$ almost surely (a.s.) as $u \rightarrow \infty$, and, further, $\mathrm{E} \tau_{u}^{S} / u \rightarrow 1 / c$ as $u \rightarrow \infty$, where $c \in(0, \infty)$ is the expected step length. These express a kind of long-term linearity of the passage time, and provide useful intuition in applications. Together with the known, classical, behaviour of other 'fluctuation' quantities related to passage over a level, such as the overshoot of the level, and various undershoots, etc., this kind of stability constitutes one of many well-known properties of the renewal theory of random walks More generally, properties such as stability of the passage time, etc., have been extended to random walks on the line. (References and further discussion are given later.)

It is natural to consider carrying the discrete-time results over to a Lévy process $\left(X_{t}\right)_{t \geq 0}$, and this has been done in the literature for some of the fluctuation quantities; see in particular [17] for stability of the overshoot. Applications of this and related kinds of result abound; we have in mind, in particular, applications to the insurance risk process; see, e.g. recent results in [5], [15], [19], [28], and [31]. These authors have tended to concentrate on properties of the overshoot and undershoots, with less attention paid to the ruin time, $\tau_{u}$. But it could be argued that $\tau_{u}$ is the most important or at least the most interesting variable, from a practical point of view.

Received 25 November 2010; revision received 28 April 2011.

* Postal address: Department of Mathematics, Syracuse University, Syracuse, NY 13244-1150, USA.

Email address: psgriffi@syr.edu

** Postal address: Centre for Financial Mathematics, and School of Finance, Actuarial Studies and Applied Statistics, Australian National University, Canberra, ACT 0200, Australia.

Research partially supported by ARC grant DP1092502. 
Our aim in this paper is to set out in detail a comprehensive listing of conditions for the stability of $\tau_{u}$ in the Lévy setting. For 'large-time' stability, i.e. as $u \rightarrow \infty$, the discretetime (random walk) results can be consulted to give useful guidance for some of the Lévy results; some are rather straightforward to transfer, but others again are challenging. We consider both stability in probability and almost-sure stability of $\tau_{u} / u$ as $u \rightarrow \infty$, both when $\lim \sup _{t \rightarrow \infty} X_{t}=\infty$ a.s. and when $\lim _{t \rightarrow \infty} X_{t}=-\infty$ a.s.

Even more interesting is the 'small-time' stability, i.e. as $u \rightarrow 0$, of the passage time. Here there are of course no corresponding random walks that can be used for guidance, but, remarkably, small-time results for Lévy processes often parallel large-time results in certain ways. With this insight and some further analysis we are able to give also a comprehensive analysis of the small-time stability of $\tau_{u}$. Some curious and unexpected results occur (see, e.g. Remark 2.3). Such results may be thought of as adding to our understanding of the local properties of Lévy processes.

The setting is as follows. Suppose that $X=\left\{X_{t}: t \geq 0\right\}, X_{0}=0$, is a Lévy process defined on $(\Omega, \mathcal{F}, P)$, with triplet $\left(\gamma, \sigma^{2}, \Pi_{X}\right), \Pi_{X}$ being the Lévy measure of $X, \gamma \in \mathbb{R}$, and $\sigma^{2} \geq 0$. Thus, the characteristic function of $X$ is given by the Lévy-Khintchine representation, $\mathrm{E}\left(\mathrm{e}^{\mathrm{i} \theta X_{t}}\right)=\mathrm{e}^{t \Psi_{X}(\theta)}$, where

$$
\Psi_{X}(\theta)=\mathrm{i} \theta \gamma-\frac{\sigma^{2} \theta^{2}}{2}+\int_{\mathbb{R}}\left(\mathrm{e}^{\mathrm{i} \theta x}-1-\mathrm{i} \theta x \mathbf{1}_{\{|x| \leq 1\}}\right) \Pi_{X}(\mathrm{~d} x) \quad \text { for } \theta \in \mathbb{R} .
$$

Denote the maximum process by

$$
\bar{X}_{t}=\sup _{0 \leq s \leq t} X_{s}
$$

and let

$$
G_{t}=\sup \left\{0 \leq s \leq t: X_{s}=\bar{X}_{s}\right\}
$$

be the time of the last maximum prior to time $t$. Our focus will be on the first passage time above level $u$, defined by

$$
\tau_{u}=\inf \left\{t \geq 0: X_{t}>u\right\}, \quad u>0 .
$$

(We adopt the convention that the inf of the empty set is $+\infty$.) Also important will be the time of the last maximum before passage, $G_{\tau_{u}-}$, and the position after transit above level $u$, $X_{\tau_{u}}$. Throughout, we assume that $\Pi_{X}$ is not identically 0 and that $X$ is not the negative of a subordinator (in which case $\tau_{u}=\infty$ for all $u>0$ ). By a compound Poisson process we will mean a Lévy process with finite Lévy measure, no Brownian component, and zero drift.

We need some further notation. Let $\left(L_{t}^{-1}, H_{t}\right)_{t \geq 0}$ denote the bivariate ascending inverse local time, ladder height subordinator process of $X$. The process $\left(L^{-1}, H\right)$ is defective when, and only when, $\lim _{t \rightarrow \infty} X_{t}=-\infty$ a.s. In that case, it is obtained from a nondefective process $\left(\mathcal{L}^{-1}, \mathscr{H}\right)$ by exponential killing with rate $q>0$, say. When $\left(L^{-1}, H\right)$ is nondefective, the killing is unnecessary and we set $\left(L^{-1}, H\right)=\left(\mathcal{L}^{-1}, \mathscr{H}\right)$ and take $q=0$. We denote the bivariate Lévy measure of $\left(\mathcal{L}_{t}^{-1}, \mathscr{H}_{t}\right)_{t \geq 0}$ by $\Pi_{L^{-1}, H}(\cdot, \cdot)$, and let $\Pi_{L^{-1}}$ and $\Pi_{H}$ be the marginal Lévy measures of $\mathcal{L}^{-1}$ and $\mathcal{H}$. The Laplace exponent $\kappa(a, b)$ of $\left(L^{-1}, H\right)$ will play an important role in our analysis. It is defined by

$$
\mathrm{e}^{-\kappa(a, b)}=\mathrm{e}^{-q} \mathrm{E}^{-a \mathscr{L}_{1}^{-1}-b \mathscr{H}_{1}}
$$


for values of $a, b \in \mathbb{R}$ for which the expectation is finite. We can write

$$
\kappa(a, b)=q+d_{L^{-1}} a+d_{H} b+\int_{t \geq 0} \int_{h \geq 0}\left(1-\mathrm{e}^{-a t-b h}\right) \Pi_{L^{-1}, H}(\mathrm{~d} t, \mathrm{~d} h),
$$

where $d_{L^{-1}} \geq 0$ and $d_{H} \geq 0$ are drift constants. See, e.g. [7, Chapter VI], [14, Chapter 4], [29, Chapter 6], and [33] for these relationships.

The following theorem connects the Laplace transform of the fluctuation quantities with the bivariate Laplace exponent. It is an extension of the 'second factorisation identity' (see [32, Equation (3.2)]). A proof of Theorem 1.1 is given in [22].

Theorem 1.1. (Laplace transform identity.) Fix $\mu>0, \rho \geq 0, \lambda \geq 0, v \geq 0$, and $\theta \geq 0$. If $\mu+\lambda \neq \rho$,

$$
\begin{aligned}
\int_{u \geq 0} & \mathrm{e}^{-\mu u} \mathrm{E}\left(\exp \left(-\rho\left(X_{\tau_{u}}-u\right)-\lambda\left(u-\bar{X}_{\tau_{u}-}\right)-\nu G_{\tau_{u}-}-\theta\left(\tau_{u}-G_{\tau_{u}-}\right)\right) ; \tau_{u}<\infty\right) \mathrm{d} u \\
& =\frac{\kappa(\theta, \mu+\lambda)-\kappa(\theta, \rho)}{(\mu+\lambda-\rho) \kappa(\nu, \mu)} .
\end{aligned}
$$

In the present paper we apply these concepts to study the stability of the passage time, $\tau_{u}$, by which we mean that $\tau_{u} / u$ has a finite and positive nonstochastic limit, where the convergence may be as $u \rightarrow 0$ or $u \rightarrow \infty$, and the convergence may be in probability, almost sure, or in mean. We will also consider, to a lesser extent, the position, $X_{\tau_{u}}$, of $X$ as it crosses the boundary. Some other results of interest, especially that the $\tau_{u} / u$ are uniformly integrable as $u \rightarrow \infty$ if $X$ has a finite positive mean (see Lemma 5.2), are derived as by-products.

The results relating to the stability of $\tau_{u}$ are given in Section 2. In contrast, in Section 3 we consider the large-time conditional stability of $\tau_{u}$ when $\mathrm{P}\left(\tau_{u}<\infty\right) \rightarrow 0$ as $u \rightarrow \infty$. This is the usual setup in the Lévy insurance risk model, for which we refer the reader to, e.g. [1], [3], [15], and [28] for background and references. Section 4 contains some concluding remarks and references. All proofs are given in Sections 5 and 6, and Appendix A.

\section{Stability}

This section contains results relating to the stability of $\tau_{u}$ as $u \rightarrow L$, where $L=\infty$ or $L=0$. For stability to make sense when $L=\infty$, we need, at a minimum, to assume that $\mathrm{P}\left(\tau_{u}<\infty\right) \rightarrow 1$ as $u \rightarrow \infty$. This is equivalent to $\lim _{\sup } \operatorname{su}_{t \rightarrow \infty} X_{t}=+\infty$ a.s., in which case $\tau_{u}<\infty$ a.s. for all $u>0$ and $\tau_{u} \rightarrow \infty$ a.s. as $u \rightarrow \infty$. The natural analogue of this condition when $L=0$ is that $\mathrm{P}\left(\tau_{u}<\infty\right) \rightarrow 1$ and $\tau_{u} \rightarrow 0$ a.s. as $u \downarrow 0$. This is equivalent to 0 being regular for $(0, \infty)$; see [8] for an analytic equivalence. Thus, the overriding assumptions throughout this section are:

- $\lim \sup _{t \rightarrow \infty} X_{t}=+\infty$ a.s. when $L=\infty$,

- 0 is regular for $(0, \infty)$ when $L=0$.

Let $\bar{\Pi}_{X}$ and $\bar{\Pi}_{X}^{ \pm}$denote the tails of $\Pi_{X}$. Thus, for $x>0$,

$$
\begin{gathered}
\bar{\Pi}_{X}^{+}(x)=\Pi_{X}\{(x, \infty)\}, \quad \bar{\Pi}_{X}^{-}(x)=\Pi_{X}\{(-\infty,-x)\}, \\
\text { and } \bar{\Pi}_{X}(x)=\bar{\Pi}_{X}^{+}(x)+\bar{\Pi}_{X}^{-}(x),
\end{gathered}
$$


and define a kind of truncated mean

$$
\begin{aligned}
A(x) & :=\gamma+\bar{\Pi}_{X}^{+}(1)-\bar{\Pi}_{X}^{-}(1)+\int_{1}^{x}\left(\bar{\Pi}_{X}^{+}(y)-\bar{\Pi}_{X}^{-}(y)\right) \mathrm{d} y \\
& =\gamma+x\left(\bar{\Pi}_{X}^{+}(x)-\bar{\Pi}_{X}^{-}(x)\right)+\int_{1<|y| \leq x} y \Pi_{X}(\mathrm{~d} y), \quad x>0 .
\end{aligned}
$$

The first theorem concerns the stability in probability of $\tau_{u}$. Consider first the stability for large times, as $u \rightarrow \infty$, i.e. the property $\tau_{u} / u \stackrel{\mathrm{P}}{\rightarrow} 1 / c$ as $u \rightarrow \infty$ for some $c \in(0, \infty)$. This is equivalent to the relative stability in probability of the process $X$ itself, i.e. to $X_{t} / t \stackrel{\mathrm{P}}{\rightarrow} c$ as $t \rightarrow \infty$. We prove it via an equivalence of the stability of $\tau_{u}$ with that of $\bar{X}$, namely, $\bar{X}_{t} / t \stackrel{\mathrm{P}}{\rightarrow} c$ as $t \rightarrow \infty$, a trivial relationship. We then show that the latter holds if and only if $X$ is relatively stable, which is not entirely obvious, but follows from similar (large-time) random walk working of [27], where the stability of the passage time of a random walk above a constant level is considered for general norming sequences. The stability of $\tau_{u}$ is connected to the bivariate Laplace exponent in (2.3) below, which is a new relationship, derived via Theorem 1.1, and the list of equivalences for this case is completed by that of (2.5) and (2.6), below, which is given in Theorem 3.1 of [16].

This list, for the case $u \rightarrow \infty, c \in(0, \infty)$, then sets the pattern we work from for the case $u \downarrow 0, c \in(0, \infty)$, and later results. Theorem 2.1 also includes the cases $c=0$ and $c=\infty$ for completeness, though these strictly speaking do not give rise to stability conditions.

Theorem 2.1. (Stability in probability of the exit time.) (a) Fix a constant $c \in(0, \infty)$, and let $L=0$ or $\infty(1 / L=\infty$ or 0$)$. Then the following statements are equivalent:

$$
\begin{aligned}
& \frac{\tau_{u}}{u} \stackrel{\mathrm{P}}{\rightarrow} \frac{1}{c} \text { as } u \rightarrow L, \\
& \lim _{x \rightarrow 1 / L} \frac{\kappa(x, 0)}{\kappa(x, \xi x)}=\frac{1}{1+\xi c} \text { for each } \xi>0, \\
& \frac{\bar{X}_{t}}{t} \stackrel{\mathrm{P}}{\rightarrow} c \text { as } t \rightarrow L, \\
& \frac{X_{t}}{t} \stackrel{\mathrm{P}}{\rightarrow} c \text { as } t \rightarrow L .
\end{aligned}
$$

In the case $L=\infty,(2.2)-(2.5)$ are equivalent to

$$
x \bar{\Pi}_{X}(x) \rightarrow 0 \text { and } A(x) \rightarrow c \text { as } x \rightarrow \infty .
$$

In the case $L=0,(2.2)-(2.5)$ are equivalent to

$$
\sigma^{2}=0, \quad x \bar{\Pi}_{X}(x) \rightarrow 0, \quad \text { and } \quad A(x) \rightarrow c, \quad \text { as } x \downarrow 0 .
$$

(b) Suppose that $c=0$. If $L=\infty$ then (2.2)-(2.6) remain equivalent. If $L=0$ then (2.2)-(2.4) remain equivalent, as do (2.5) and (2.7). However, while (2.5) implies (2.2)-(2.4), the converse does not hold when $L=0$.

(c) Suppose that $c=\infty$. Then (2.2)-(2.4) remain equivalent for $L=0$ or $\infty$ in the following sense:

$$
\begin{aligned}
\frac{\tau_{u}}{u} \stackrel{\mathrm{P}}{\rightarrow} 0 \quad \text { as } u \rightarrow L & \Longleftrightarrow \lim _{x \rightarrow 1 / L} \frac{\kappa(x, 0)}{\kappa(x, \xi x)}=0 \quad \text { for each } \xi>0 \\
& \Longleftrightarrow \frac{\bar{X}_{t}}{t} \stackrel{\mathrm{P}}{\rightarrow} \infty \text { as } t \rightarrow L .
\end{aligned}
$$

Again, while (2.5) implies (2.2)-(2.4), it is not equivalent in either case, $L=0$ or $\infty$. 
Remark 2.1. As mentioned above, the equivalence of (2.5) and (2.6) when $L=\infty$ is given in [16], while the equivalence of (2.5) and (2.7) when $L=0$ is given in Theorem 3.1 of [16]. Both of these results hold for all $c \in(-\infty, \infty)$. We include them in the statement of Theorem 2.1 for completeness and for the convenience of the reader.

The next theorem concerns the almost-sure stability of $\tau_{u}$. We follow the pattern set by Theorem 2.1. The connection with the bivariate Laplace exponent is transmuted in this case to requiring finite first moments of the ladder processes $H$ and $L^{-1}$. Almost-sure stability for large times requires a finite positive mean for $X$ (for large times), and bounded variation with positive drift of $X$ (for small times). Recall that when $X$ is of bounded variation, we may write the Lévy-Khintchine exponent in the form

$$
\Psi(\theta)=\mathrm{i} \theta d_{X}+\int_{\mathbb{R}}\left(\mathrm{e}^{\mathrm{i} \theta x}-1\right) \Pi_{X}(\mathrm{~d} x),
$$

where $d_{X}=\gamma-\int x \mathbf{1}_{\{|x| \leq 1\}} \Pi_{X}(\mathrm{~d} x)$ is called the drift of $X$.

Theorem 2.2. (Almost-sure stability of the exit time.) (a) Fix $c \in[0, \infty)$.

(i) We have $\tau_{u} / u \rightarrow 1 / c$ a.s. as $u \rightarrow \infty$ if and only if $\mathrm{E}\left|X_{1}\right|<\infty$ and $\mathrm{E} X_{1}=c \geq 0$.

(ii) We have $\tau_{u} / u \rightarrow 1 / c$ a.s. as $u \rightarrow 0$ if and only if $X$ is of bounded variation with drift $d_{X}=c \geq 0$.

(b) Fix $c \in(0, \infty)$. Then (i) holds if and only if $\mathrm{E} H_{1}<\infty$ and $\mathrm{E} L_{1}^{-1}<\infty$, in which case $c=\mathrm{E} H_{1} / \mathrm{E} L_{1}^{-1}$, while (ii) holds if and only if $\sigma^{2}=0, d_{L^{-1}}>0$, and $d_{H}>0$, in which case $c=d_{H} / d_{L^{-1}}$.

Remark 2.2. Note that, respectively under (2.6) and (2.7),

$$
\lim _{x \rightarrow \infty} A(x)=\gamma+\int_{|y|>1} y \Pi_{X}(\mathrm{~d} y) \quad \text { and } \quad \lim _{x \downarrow 0} A(x)=\gamma-\int_{0<|y| \leq 1} y \Pi_{X}(\mathrm{~d} y) .
$$

Here existence of the limits is equivalent to conditional convergence of the integrals. Under the conditions of Theorem 2.2(a)(i) and (a)(ii), these integrals converge absolutely and the limits are then given by $\mathrm{E} X_{1}$ and $d_{X}$, respectively, thus confirming that the expressions for $c$ in Theorems 2.1 and 2.2 agree. The difference between (2.6) and (2.7) and Theorem 2.2(i) and (ii) is essentially whether the integrals in (2.9) converge conditionally or absolutely.

In the next theorem we examine the convergence of $\mathrm{E} \tau_{u} / u$ as $u \rightarrow \infty$ and as $u \downarrow 0$. Recall that $\mathrm{E} \tau_{u}<\infty$ for some, hence all, $u \geq 0$ if and only if $X$ drifts to $+\infty$ a.s., if and only if E $L_{1}^{-1}<\infty$ (see, e.g. Theorem 1 of [18]).

Theorem 2.3. (Stability of the expected exit time.) (a) Fix $c \in(0, \infty)$. Then the following statements hold.

(i) $\mathrm{E} \tau_{u}<\infty$ for each $u>0$ and $\lim _{u \rightarrow \infty} \mathrm{E} \tau_{u} / u=1 /$ c if and only if $0<\mathrm{E} X_{1} \leq \mathrm{E}\left|X_{1}\right|<$ $\infty$. In this situation, $\mathrm{E} H_{1}<\infty, \mathrm{E} L_{1}^{-1}<\infty$, and $c=\mathrm{E} X_{1}=\mathrm{E} H_{1} / \mathrm{E} L_{1}^{-1}$.

(ii) $\mathrm{E} \tau_{u}<\infty$ for each $u>0$ and $\lim _{u \downarrow 0} \mathrm{E} \tau_{u} / u=1 / c$ if and only if $\mathrm{E} L_{1}^{-1}<\infty$ and $d_{H}>0$, and then $c=d_{H} / \mathrm{E} L_{1}^{-1}$. 
(b) Consider the case $c=0$.

(i) In part (a)(i) of the theorem, the case $c=0$ cannot arise; when $\mathrm{E} \tau_{u}<\infty$ for each $u>0, \lim _{u \rightarrow \infty} \mathrm{E} \tau_{u} / u$ exists and is in $[0, \infty)$.

(ii) We have $\mathrm{E} \tau_{u}<\infty$ for each $u>0$ and $\lim _{u \downarrow 0} \mathrm{E} \tau_{u} / u=\infty$ if and only if $\mathrm{E} L_{1}^{-1}<\infty$ and $d_{H}=0$.

(c) Consider the case $c=\infty$.

(i) We have $\mathrm{E} \tau_{u}<\infty$ for each $u>0$ and $\lim _{u \rightarrow \infty} \mathrm{E} \tau_{u} / u=0$ if and only if $\mathrm{E} L_{1}^{-1}<\infty$ and $\mathrm{E} H_{1}=\infty$.

(ii) In part (a)(ii) of the theorem, the case $c=\infty$ cannot arise; when $\mathrm{E} \tau_{u}<\infty$ for each $u>0$, we always have $\liminf _{u \downarrow 0} \mathrm{E} \tau_{u} / u>0$.

Remark 2.3. (i) It is curious that the formula for $c$ in Theorem 2.3(a)(ii) does not agree with the corresponding versions in Theorem 2.1 or Theorem 2.2. We give an example to illustrate how the difference can arise. Let

$$
X_{t}=a t-N_{t},
$$

where $N_{t}$ is a rate-one Poisson process and $a>1$. Thus, $\lim _{t \rightarrow \infty} X_{t}=\infty$ a.s. Since $\tau_{u}=u a^{-1}$ for sufficiently small $u$, it trivially follows that

$$
\lim _{u \downarrow 0} \frac{\tau_{u}}{u}=\frac{1}{a} \quad \text { a.s. }
$$

We claim that

$$
\frac{\mathrm{E} \tau_{u}}{u} \rightarrow \frac{1+\mathrm{E} \tau_{1}}{a} .
$$

This is because if $\xi$ is the time of the first jump of $N$ then

$$
\begin{aligned}
\mathrm{E} \tau_{u} & =\mathrm{E}\left(\tau_{u} ; N_{u a^{-1}}=0\right)+\mathrm{E}\left(\tau_{u} ; N_{u a^{-1}} \geq 1\right) \\
& =u a^{-1} \mathrm{e}^{-u a^{-1}}+\int_{0}^{u a^{-1}} \mathrm{E}\left(\tau_{u} \mid \xi=t\right) \mathrm{P}(\xi \in \mathrm{d} t) \\
& =u a^{-1} \mathrm{e}^{-u a^{-1}}+\int_{0}^{u a^{-1}}\left(t+\mathrm{E} \tau_{1-a t+u}\right) \mathrm{e}^{-t} \mathrm{~d} t
\end{aligned}
$$

Since $\tau_{1+x} \stackrel{\mathrm{P}}{\rightarrow} \tau_{1}$ as $x \downarrow 0,(2.10)$ now follows after dividing by $u$ and taking the limit.

We now check that this agrees with Theorem 2.3(a)(ii). For the normalization of $L$, the local time at the maximum, we take

$$
L_{t}=\int_{0}^{t} \mathbf{1}_{\left\{X_{s}=\bar{X}_{s}\right\}} \mathrm{d} s .
$$

Then the ladder height process is linear drift, $H_{t}=a t$. Hence, $d_{H}=a$. By construction,

$$
L_{t}^{-1}=t+\sum_{1}^{N_{t}} R_{i},
$$

where again $N_{t}$ is a rate-one Poisson process and $R_{i}$ are independent and identically distributed (i.i.d.) random variables independent of $N$, with distribution the same as that of $\tau_{1}$. Hence, $d_{L^{-1}}=1$ and

$$
\mathrm{E} L_{1}^{-1}=1+\mathrm{E} \tau_{1}
$$


so the $c$ in Theorem 2.3(a)(ii) is $d_{H} / \mathrm{E} L_{1}^{-1}=a /\left(1+\mathrm{E} \tau_{1}\right)$, giving agreement with (2.10). On the other hand, the $c$ in Theorem 2.2(a)(ii) is $d_{X}=d_{H} / d_{L^{-1}}=a$.

We now turn to the stability of the time of the last maximum before ruin. As may be expected, this is a more difficult object to study than $\tau_{u}$. We consider the three modes of convergence investigated in Theorems 2.1-2.3.

Theorem 2.4. (Stability of the last maximum before ruin.) Let $L=0$ or $\infty(1 / L=\infty$ or $0)$.

(a) Fix $c \in(0, \infty)$. We have

$$
\frac{G_{\tau_{u}-}}{u} \stackrel{\mathrm{P}}{\rightarrow} \frac{1}{c} \text { as } u \rightarrow L
$$

if and only if

$$
\lim _{x \rightarrow 1 / L} \frac{\kappa(0, x)}{\kappa(\xi x, x)}=\frac{c}{c+\xi} \quad \text { for each } \xi>0
$$

(b) Fix $c \in[0, \infty)$.

(i) $G_{\tau_{u}-} / u \rightarrow 1 / c$ a.s. as $u \rightarrow \infty$ if and only if $\mathrm{E}\left|X_{1}\right|<\infty$ and $\mathrm{E} X_{1}=c \geq 0$.

(ii) $G_{\tau_{u}-} / u \rightarrow 1 / c$ a.s. as $u \rightarrow 0$ if and only if $X$ is of bounded variation with drift $d_{X}=c \geq 0$.

(c) If $0<\mathrm{E} X_{1}<\infty$ then $\lim _{u \rightarrow \infty} \mathrm{E} G_{\tau_{u}-} / u=1 / \mathrm{E} X_{1}$.

Remark 2.4. It is not clear how the conditions of Theorem 2.1 relate to the stability in probability of $G_{\tau_{u}-}$. We can show that (2.2)-(2.6) imply (2.11) and (2.12), but it is not clear whether or not the converse holds. For almost-sure convergence, the results for $G_{\tau_{u}}$ parallel those for $\tau_{u}$. For convergence in mean, the situation remains largely unresolved.

The final result, Theorem 2.5, belongs in the present section since it holds in the case when $\lim _{t \rightarrow \infty} X_{t}=+\infty$ a.s., but we apply it in the next section, in the case when $\lim _{t \rightarrow \infty} X_{t}=-\infty$ a.s., to obtain results in the Lévy insurance risk model.

Theorem 2.5. (Convergence of expected exit times with overshoot.) Assume that $0<\mathrm{E} X_{1} \leq$ $\mathrm{E}\left|X_{1}\right|<\infty$, and that $X$ is not compound Poisson, or is compound Poisson with a nonlattice jump distribution. Then, for all $\rho>0$,

$$
\lim _{u \rightarrow \infty} \mathrm{E}\left(\frac{G_{\tau_{u}-}}{u} \mathrm{e}^{-\rho\left(X_{\tau_{u}}-u\right)}\right)=\lim _{u \rightarrow \infty} \mathrm{E}\left(\frac{\tau_{u}}{u} \mathrm{e}^{-\rho\left(X_{\tau_{u}}-u\right)}\right)=\frac{1}{\mathrm{E} X_{1}} \mathrm{E}^{-\rho Y},
$$

where $Y$ is a random variable having the limiting distribution of the overshoot $X_{\tau_{u}}-u$, and has density $\bar{\Pi}_{H}(h) \mathrm{d} h / \mathrm{E} H_{1}$ on $(0, \infty)$ and mass $d_{H} / \mathrm{E} H_{1}$ at 0 .

\section{Stability in the insurance risk model}

The aim of this section is to illustrate that stability questions are also of interest when $X_{t} \rightarrow-\infty$ a.s. as $t \rightarrow \infty$. We phrase the discussion in terms of an insurance risk model. In this case $X$ represents the excess in claims over premium of an insurance company. The classical model in this context is the Cramér-Lundberg model in which $X$ is the sum of a compound Poisson process with positive jumps, representing claims, and a negative drift, representing premium inflow. The results in the present section will be given for a general Lévy insurance risk model where no such restrictions are placed on $X$. 
The over-riding assumption throughout this section is the Cramér condition, namely, that

$$
\mathrm{Ee}^{\nu_{0} X_{1}}=1 \text { for some } v_{0}>0 \text {. }
$$

It is well known that, under (3.1), $\mathrm{E} X_{1}$ is well defined, with $\mathrm{E} X_{1}^{+}<\infty, \mathrm{E} X_{1}^{-} \in(0, \infty]$, and $\mathrm{E} X_{1} \in[-\infty, 0)$, and so $\lim _{t \rightarrow \infty} X_{t}=-\infty$ a.s. Furthermore, $\mathrm{E}\left(X_{1} \mathrm{e}^{\nu X_{1}}\right)$ is finite and positive for all $v$ in a left neighbourhood of $v_{0}$, and

$$
\mu^{*}:=\mathrm{E}\left(X_{1} \mathrm{e}^{\nu_{0} X_{1}}\right)>0 \quad\left(\text { possibly } \mu^{*}=+\infty\right) .
$$

Since $\lim _{t \rightarrow \infty} X_{t}=-\infty$ a.s., we are in the situation that $\mathrm{P}\left(\tau_{u}<\infty\right)<1$ for all $u>0$, and $\lim _{u \rightarrow \infty} \mathrm{P}\left(\tau_{u}<\infty\right)=0$. In an insurance risk context, we are interested in forecasting the ruin time $\tau_{u}$ in a worst case scenario, i.e. conditional on $\tau_{u}<\infty$ ('ruin occurs'). Asymptotic properties of $\tau_{u}$ and associated variables, conditional on $\tau_{u}<\infty$, often provide surprisingly good approximations of corresponding finite-level distributions; cf, e.g. [20, Appendix]. In the present context we look at the stability of $\tau_{u}, G_{\tau_{u}-}$, and $X_{\tau_{u}}$, showing that they are asymptotically linear under mild conditions.

We need some more infrastructure. Let $\left(X_{t}^{*}\right)_{t \geq 0}$ denote the Esscher transform of $X$ defined by

$$
\mathrm{P}\left(\left(X_{s}^{*}, 0 \leq s \leq t\right) \in B, X_{t}^{*} \in \mathrm{d} x\right)=\mathrm{e}^{\nu_{0} x} \mathrm{P}\left(\left(X_{s}, 0 \leq s \leq t\right) \in B, X_{t} \in \mathrm{d} x\right)
$$

for any Borel subset $B$ of $\mathbb{R}^{[0, t]}$. Equivalently, $X^{*}$ may be introduced by means of exponential tilting; that is, define a new probability $\mathrm{P}^{*}$, given on $\widetilde{F}_{t}$ by

$$
\frac{\mathrm{dP}^{*}}{\mathrm{dP}}=\mathrm{e}^{v_{0} X_{t}}
$$

Then $X$ under $\mathrm{P}^{*}$ has the same distribution as $X^{*}$ under $\mathrm{P}$. It easily follows that

$$
\mathrm{E} f\left(X_{t}^{*}\right)=\mathrm{E}^{*} f\left(X_{t}\right)=\mathrm{E}\left(f\left(X_{t}\right) \mathrm{e}^{\nu_{0} X_{t}}\right)
$$

for any Borel function $f$ for which the expectations are finite. Here $X^{*}$ is itself a Lévy process with exponent $\Psi\left(\theta-\mathrm{i} \nu_{0}\right)$ and $\mathrm{E}^{*} X_{1}=\mu^{*}$. Since $\mu^{*}>0$ by (3.2), $X_{t}^{*}$ drifts to $+\infty$ a.s., and, hence, $\left(H_{t}^{*}\right)_{t \geq 0}$, the increasing ladder height process associated with $\left(X_{t}^{*}\right)_{t \geq 0}$, is proper.

Our setup is that of Bertoin and Doney [9]. The main result in [9], which we give in the form proved in Theorem 7.6 of [29] (see also Section XIII.5 of [4]), is as follows.

Theorem 3.1. ([9].) Suppose that (3.1) holds and that the support of $\Pi_{X}$ is nonlattice in the case that $X$ is compound Poisson. Then

$$
\lim _{u \rightarrow \infty} \mathrm{e}^{v_{0} u} \mathrm{P}\left(\tau_{u}<\infty\right)=C \in[0, \infty)
$$

where $C:=\mathrm{E}^{*} \mathrm{e}^{-v_{0} Y}>0$ if and only if $\mu^{*}<\infty$. Here $Y$ is a random variable having the limiting distribution of the overshoot $X_{\tau_{u}}-u$ under $\mathrm{P}^{*}$.

To state the stability result for the general Lévy insurance risk model under (3.1), introduce the probability measure $\mathrm{P}^{(u)}(\cdot)=\mathrm{P}\left(\cdot \mid \tau_{u}<\infty\right)$, and denote convergence in probability conditional on $\tau_{u}<\infty$ by ' $\stackrel{\mathrm{P}^{(u)}}{\longrightarrow}$ '. 
Theorem 3.2. Assume that (3.1) holds and that $\mu^{*}<\infty$ (so that $0<\mu^{*}<\infty$ ). Then, as $u \rightarrow \infty$,

$$
\frac{X_{\tau_{u}}}{u} \stackrel{\mathrm{p}^{(u)}}{\longrightarrow} 1, \quad \frac{G_{\tau_{u}-}}{u} \stackrel{\mathrm{p}^{(u)}}{\longrightarrow} \frac{1}{\mu^{*}}, \quad \text { and } \quad \frac{\tau_{u}}{u} \stackrel{\mathrm{p}^{(u)}}{\longrightarrow} \frac{1}{\mu^{*}} .
$$

Assume in addition that the support of $\Pi_{X}$ is nonlattice in the case that $X$ is compound Poisson. Then

$$
\lim _{u \rightarrow \infty} \frac{\mathrm{E}^{(u)} X_{\tau_{u}}}{u}=1, \quad \lim _{u \rightarrow \infty} \frac{\mathrm{E}^{(u)} G_{\tau_{u}-}}{u}=\frac{1}{\mu^{*}}, \quad \text { and } \quad \lim _{u \rightarrow \infty} \frac{\mathrm{E}^{(u)} \tau_{u}}{u}=\frac{1}{\mu^{*}} .
$$

Parts of our Theorem 3.2 are well known for the Cramér-Lundberg model, and their extension to the general Lévy insurance risk model is straightforward. Others appear to be new.

\section{Concluding remarks}

There is of course a very large literature on (large-time) renewal theorems for random walks, and, more recently, some similar results have been proved for Lévy processes. Regarding the ruin time, most results so far concern the infinite horizon ruin probability, $\mathrm{P}\left(\tau_{u}<\infty\right)$, or, equivalently, the distribution of the overall maximum of the random walk or Lévy process, and we do not attempt to summarise them here (other than the references mentioned in Sections 1-3). A web search turns up many such papers and books.

The finite horizon ruin probability, $\mathrm{P}\left(\tau_{u}<T\right)$, is less studied, but important results are obtained in, e.g. [2], [5], [6], [12], [25], [26] (see also their references), and especially in the insurance/actuarial literature (usually from a more applied point of view). These results of course give information on the long-run distribution of the ruin time, conditional on ruin occurring. A more recent result along these lines is given in [21], assuming, like [12] and [25], convolution equivalent conditions on the tails of the process or its Lévy measure. These authors are interested in the asymptotic distribution of $\tau_{u}$, rather than in its stability per se; as mentioned earlier, results on stability such as we give are more akin to classical (large-time) renewal theory than to these, and small-time versions, which make sense for Lévy processes but not for random walks, have previously been neglected, in the main.

We turn now to the proofs.

\section{Proofs for Section 2}

We assume throughout this section that $\limsup _{t \rightarrow \infty} X_{t}=+\infty$ a.s. when $L=\infty$, and 0 is regular for $(0, \infty)$ when $L=0$. In the former case, $\tau_{u}<\infty$ a.s. for all $u>0$, while $\mathrm{P}\left(\tau_{u}<\infty\right) \rightarrow 1$ as $u \rightarrow 0$ in the latter case.

Proof of Theorem 2.1. We first prove parts (a) and (b). Let $c \in[0, \infty)$ until further notice, with the obvious interpretations when $c=0$. Assume that (2.2) holds with $u \rightarrow L$. From (1.2) we have, for $u>0$ and $\theta>0$,

$$
\mu \int_{u \geq 0} \mathrm{e}^{-\mu u} \mathrm{E}\left(\mathrm{e}^{-\theta \tau_{u}} ; \tau_{u}<\infty\right) \mathrm{d} u=1-\frac{\kappa(\theta, 0)}{\kappa(\theta, \mu)} .
$$

Take $y>0$, and replace $\mu$ by $\mu / y, u$ by $u y$, and $\theta$ by $\theta / y$ in (5.1) to get

$$
\mu \int_{u \geq 0} \mathrm{e}^{-\mu u} \mathrm{E}\left(\mathrm{e}^{-\theta \tau_{u y} / y} ; \tau_{u y}<\infty\right) \mathrm{d} u=1-\frac{\kappa(\theta / y, 0)}{\kappa(\theta / y, \mu / y)} .
$$


By hypothesis, $\tau_{u y} / y \stackrel{\mathrm{P}}{\rightarrow} u / c$ as $y \rightarrow L$ for each $u>0$, so letting $y \rightarrow L$ in (5.2) gives, by dominated convergence,

$$
\lim _{y \rightarrow L} \frac{\kappa(\theta / y, 0)}{\kappa(\theta / y, \mu / y)}=1-\mu \int_{u \geq 0} \mathrm{e}^{-\mu u} \mathrm{e}^{-\theta u / c} \mathrm{~d} u=\frac{\theta}{\theta+\mu c} .
$$

Replacing $y$ by $x=\theta / y \rightarrow 1 / L$ and $\mu / \theta$ by $\xi$ gives (2.3) with $x \rightarrow 1 / L$.

Conversely, assume that (2.3) holds with $x \rightarrow 1 / L$. Then from (5.2) we see that, for $\theta>0$,

$$
\lim _{y \rightarrow L} \mu \int_{u \geq 0} \mathrm{e}^{-\mu u} \mathrm{E}\left(\mathrm{e}^{-\theta \tau_{u y} / y} ; \tau_{u y}<\infty\right) \mathrm{d} u=\frac{\mu c}{\theta+\mu c} .
$$

For each $y>0$ and $\theta>0$, the function $f_{y}(u, \theta):=\mathrm{E}\left(\mathrm{e}^{-\theta \tau_{u y} / y} ; \tau_{u y}<\infty\right)$ is monotone decreasing in $u$ and bounded by 1 . Given any sequence $y_{k} \rightarrow L$, we can, by Helly's theorem, find a subsequence $\tilde{y}_{k} \rightarrow L$, possibly depending on $\theta$ but not on $u$, such that $f_{\widetilde{y}_{k}}(u, \theta) \rightarrow$ $\widetilde{f}(u, \theta)$ for some function $\widetilde{f}(u, \theta) \in[0,1]$. Then by dominated convergence we have

$$
\mu \int_{u \geq 0} \mathrm{e}^{-\mu u} \tilde{f}(u, \theta) \mathrm{d} u=\frac{\mu c}{\theta+\mu c}=\mu \int_{u \geq 0} \mathrm{e}^{-\mu u} \mathrm{e}^{-\theta u / c} \mathrm{~d} u,
$$

and from the uniqueness of Laplace transforms we deduce that $\tilde{f}(u, \theta)=\mathrm{e}^{-\theta u / c}$, not dependent on the choice of subsequence. Hence (taking $u=1$ now),

$$
\lim _{y \rightarrow L} \mathrm{E}\left(\mathrm{e}^{-\theta \tau_{y} / y} ; \tau_{y}<\infty\right)=\mathrm{e}^{-\theta / c}, \quad \theta>0,
$$

proving (2.2) with $u \rightarrow L$.

Since

$$
\left\{\tau_{u}>t\right\} \subseteq\left\{\bar{X}_{t} \leq u\right\} \subseteq\left\{\tau_{u} \geq t\right\}, \quad t>0, u>0,
$$

we easily see that (2.2) is equivalent to (2.4) in either case, $L=\infty$ or $L=0$, for $c \geq 0$.

Next we show that (2.5) implies (2.4). First consider the case $L=\infty$. By Theorem 3.1 of [16], (2.5) with $t \rightarrow \infty$ is equivalent to (2.6), and the first relation in (2.6) implies that

$$
\lim _{x \rightarrow \infty} \frac{V(x)}{x}=0
$$

where

$$
V(x):=\sigma^{2}+\int_{|y| \leq x} y^{2} \Pi_{X}(\mathrm{~d} y) .
$$

To deduce (2.4) from (2.6) and (5.4) in the case $L=\infty$, decompose $X$ into small and large jump components as in Lemma 6.1 of [16] to get

$$
X_{s}=s v(t)+\sigma B_{s}+X_{s}^{(1)}+X_{s}^{(2)}, \quad 0 \leq s \leq t,
$$

where

$$
v(x):=\gamma+\int_{1<|y| \leq x} y \Pi_{X}(\mathrm{~d} y), \quad x>0,
$$

$\sigma B_{S}+X_{S}^{(1)}$ is a mean 0 martingale with jumps bounded in modulus by $t$ and all moments finite, and

$$
X_{s}^{(2)}=\sum_{0<r \leq s} \Delta X_{r} \mathbf{1}_{\left\{\left|\Delta X_{r}\right|>t\right\}}
$$


By Doob's inequality, for $\varepsilon>0$,

$$
\mathrm{P}\left(\sup _{0 \leq s \leq t}\left|\sigma B_{s}+X_{s}^{(1)}\right|>\varepsilon t\right) \leq \frac{1}{(\varepsilon t)^{2}} \mathrm{E}\left(\sigma B_{t}+X_{t}^{(1)}\right)^{2}=\frac{1}{\varepsilon^{2} t} V(t),
$$

and this tends to 0 as $t \rightarrow \infty$ by (5.4). Also,

$$
\mathrm{P}\left(\sup _{0 \leq s \leq t}\left|X_{s}^{(2)}\right|=0\right) \geq \mathrm{P}\left(\text { no jumps with }\left|\Delta X_{s}\right|>t \text { occur by time } t\right)=\exp \left(-t \bar{\Pi}_{X}(t)\right) \rightarrow 1
$$

as $t \rightarrow \infty$, while

$$
v(t)=A(t)-t \bar{\Pi}^{+}(t)+t \bar{\Pi}^{-}(t) \rightarrow c,
$$

both by (2.6). Thus, we have $\mathrm{P}\left(\sup _{0 \leq s \leq t}\left|X_{s}-c s\right|>\varepsilon t\right) \rightarrow 0$ as $t \rightarrow \infty$, which implies (2.4) with $L=\infty$ and $c \geq 0$.

Now we deal with the implication $(2.5) \Rightarrow(2.4)$ in the case $L=0$. Note that, by Theorem 2.1 of [16], (2.5) with $t \downarrow 0$ is equivalent to (2.7), and the first and second relations in (2.7) imply that

$$
\frac{V(x)}{x}=\frac{2}{x} \int_{0}^{x} y \bar{\Pi}_{X}(y) \mathrm{d} y+x \bar{\Pi}_{X}(x)=\frac{2}{x} \int_{0}^{x} o(1) \mathrm{d} y+o(1)=o(1) \quad \text { as } x \downarrow 0 .
$$

This takes the place of (5.4) in the $L=0$ case, and the rest of the proof that (2.5) implies (2.4) with $L=0$ is virtually the same as for the case with $t \rightarrow \infty$.

We have left to show that (2.4) implies (2.5) except when $c=L=0$. This is obvious if $c=0$ and $L=\infty$, so suppose that $c>0$. Note that, for $t>0$,

$$
\bar{X}_{2 t}=\bar{X}_{t} \vee\left(\sup _{t<s \leq 2 t} X_{s}\right)=\bar{X}_{t} \vee\left(X_{t}+\sup _{t<s \leq 2 t}\left(X_{s}-X_{t}\right)\right)=\bar{X}_{t} \vee\left(X_{t}+\bar{X}_{t}^{\prime}\right),
$$

where $\bar{X}_{t}^{\prime}$ is an independent copy of $\bar{X}_{t}$. Consequently, for $\varepsilon \in(0, c / 3)$, as $t \rightarrow L$,

$$
\begin{aligned}
o(1) & =\mathrm{P}\left(\bar{X}_{2 t} \leq(c-\varepsilon) 2 t\right) \\
& =\mathrm{P}\left(\bar{X}_{t} \vee\left(X_{t}+\bar{X}_{t}^{\prime}\right) \leq(c-\varepsilon) 2 t\right) \\
& \geq \mathrm{P}\left(\bar{X}_{t} \leq(c-\varepsilon) 2 t, X_{t}+(c+\varepsilon) t \leq(c-\varepsilon) 2 t,\left|\bar{X}_{t}^{\prime}-c t\right| \leq \varepsilon t\right) \\
& =\mathrm{P}\left(\frac{\bar{X}_{t}}{t} \leq 2(c-\varepsilon), \frac{X_{t}}{t} \leq c-3 \varepsilon\right) \mathrm{P}\left(\left|\frac{\bar{X}_{t}}{t}-c\right| \leq \varepsilon\right) \\
& \geq\left(\mathrm{P}\left(\frac{X_{t}}{t} \leq c-3 \varepsilon\right)-\mathrm{P}\left(\frac{\bar{X}_{t}}{t}>2(c-\varepsilon)\right)\right)(1+o(1)) \\
& =\left(\mathrm{P}\left(\frac{X_{t}}{t} \leq c-3 \varepsilon\right)-o(1)\right)(1+o(1)) .
\end{aligned}
$$

This shows that $\mathrm{P}\left(X_{t}>(c-3 \varepsilon) t\right) \rightarrow 1$, and since also

$$
\mathrm{P}\left(\frac{X_{t}}{t} \leq c+\varepsilon\right) \geq \mathrm{P}\left(\frac{\bar{X}_{t}}{t} \leq c+\varepsilon\right) \rightarrow 1,
$$

we have $X_{t} / t \stackrel{\text { P }}{\rightarrow} c$. Hence, (2.4) implies (2.5) for $L=0$ or $L=\infty$. 
Now we turn to the proof of part (c). The equivalences in (2.8) follow by the same methods as used in part (a), and, clearly, (2.5) in the case $c=\infty$ implies (2.4) in the case $c=\infty$.

All that remains is to give counterexamples showing that (2.4) does not imply (2.5) when $c=L=0$ or when $c=\infty$ and $L=0$ or $\infty$, which we do in Lemma 5.1 below, thus completing the proof of Theorem 2.1.

Lemma 5.1. There is a Lévy process for which 0 is regular for $(0, \infty)$ and with

$$
\frac{\bar{X}_{t}}{t} \stackrel{\mathrm{P}}{\rightarrow} 0 \quad \text { as } t \downarrow 0, \quad \text { but with } \quad \frac{X_{t}}{t} \stackrel{\mathrm{P}}{\rightarrow}-1 \quad \text { as } t \downarrow 0 .
$$

There is also a Lévy process with

$$
\frac{\bar{X}_{t}}{t} \stackrel{\mathrm{P}}{\rightarrow} \infty \quad \text { as } t \rightarrow L, \quad \text { but with } \quad \frac{X_{t}}{t} \stackrel{\mathrm{P}}{\rightarrow}-\infty \quad \text { as } t \rightarrow L,
$$

for $L=0$ or $L=\infty$.

Proof. See Appendix A.

Proof of Theorem 2.2. By a simple pathwise argument using (5.3), it easily follows that, for $L=\infty$ or $L=0$, and $c \in[0, \infty]$, we have $\lim _{u \rightarrow L} \tau_{u} / u=1 / c$ a.s. if and only if

$$
\lim _{t \rightarrow L} \frac{\bar{X}_{t}}{t}=c \quad \text { a.s. }
$$

(a)(i) If $\mathrm{E}\left|X_{1}\right|<\infty$ and $c=\mathrm{E} X_{1} \geq 0$, then (5.7) with $L=\infty$ holds by the strong law. Conversely, by Theorem 15 of [14], (5.7) implies that at least one of $\mathrm{E} X_{1}^{+}$or $\mathrm{E} X_{1}^{-}$is finite or else $X_{t} \rightarrow-\infty$. Since $\lim \sup _{t \rightarrow \infty} X_{t}=\infty$, the latter possibility is ruled out and so is the possibility that $\mathrm{E} X_{1}^{-}=\infty$. Since $c \in[0, \infty)$, the strong law and (5.7) then force $\mathrm{E}\left|X_{1}\right|<\infty$ and $c=\mathrm{E} X_{1} \geq 0$.

(a)(ii) If $X$ is of bounded variation with $d_{X} \geq 0$ then $X_{t} / t \rightarrow d_{X}$ a.s. as $t \downarrow 0$ by Theorem 39 of [14]. Hence, (5.7) holds with $c=d_{X}$. Conversely, by the same result, (5.7) implies that $X$ is of bounded variation and necessarily $d_{X} \geq 0$ since 0 is regular for $(0, \infty)$. It then follows that $c=d_{X} \geq 0$.

(b)(i) When $0<\mathrm{E} X_{1} \leq \mathrm{E}\left|X_{1}\right|<\infty$, we have $\mathrm{E} L_{1}^{-1}<\infty$ (see, e.g. Theorem 1 of [18]). Thus, letting $t \rightarrow \infty$ in

$$
\frac{X_{L_{t}^{-1}}}{L_{t}^{-1}}=\frac{H_{t}}{L_{t}^{-1}}=\frac{H_{t}}{t} \frac{t}{L_{t}^{-1}},
$$

and using the strong law, we obtain $H_{t} / t \rightarrow \mathrm{E} X_{1} \mathrm{E} L_{1}^{-1}$ as $t \rightarrow \infty$. This implies that $\mathrm{E} H_{1}<$ $\infty$ and $\mathrm{E} X_{1}=\mathrm{E} H_{1} / \mathrm{E} L_{1}^{-1}$. Conversely, $\mathrm{E} H_{1}<\infty$ implies that $0 \leq \mathrm{E} X_{1} \leq \mathrm{E}\left|X_{1}\right|<\infty$ by Theorem 8 of [17], and $\mathrm{E} L_{1}^{-1}<\infty$ implies that $X$ drifts to $+\infty$ a.s. by Theorem 1 of [18], so in fact $0<\mathrm{E} X_{1} \leq \mathrm{E}\left|X_{1}\right|<\infty$.

(b)(ii) When $X$ is of bounded variation, then $\sigma^{2}=0$, and by taking limits as $t \downarrow 0$ in (5.8), we obtain

$$
d_{L^{-1}} d_{X}=d_{H} .
$$

When $d_{X}>0, X_{t} / t \rightarrow d_{X}>0$ and $\tau_{u} / u \rightarrow 1 / d_{X}<\infty$ a.s. (by (a)(ii)). Thus,

$$
\frac{X_{\tau_{u}}}{u}=\frac{X_{\tau_{u}}}{\tau_{u}} \frac{\tau_{u}}{u} \rightarrow 1 \quad \text { a.s. as } u \downarrow 0 .
$$


This implies that $d_{H}>0$ by Theorem 4 of [17]. Hence, by (5.9), $d_{L^{-1}}>0$ also, and so $c=d_{X}=d_{H} / d_{L^{-1}}$.

Conversely, assume that $d_{H}>0, d_{L^{-1}}>0$, and $\sigma^{2}=0$. We show that $\lim _{u \downarrow 0} \tau_{u} / u=$ $d_{L^{-1}} / d_{H}$ a.s. Let $T_{u}:=\inf \left\{t>0: H_{t}>u\right\}, u>0$. Then $\tau_{u}=L_{T_{u}}^{-1}$. Hence, by (5.8),

$$
\frac{X_{\tau_{u}}}{u} \frac{u}{\tau_{u}}=\frac{H_{T_{u}}}{T_{u}} \frac{T_{u}}{L_{T_{u}}^{-1}} \rightarrow \frac{d_{H}}{d_{L}^{-1}} .
$$

Since $\lim _{u \rightarrow 0} X_{\tau_{u}} / u=1$ a.s. when $d_{H}>0$ by Theorem 4 of [17], the result follows.

Proof of Theorem 2.3. We begin by recalling that, from Theorem 1 of [18], E $\tau_{u}<\infty$ for some, hence all, $u \geq 0$, if and only if $X$ drifts to $+\infty$ a.s., if and only if $\mathrm{E} L_{1}^{-1}<\infty$.

Use identity (8) of $[7$, p. 174] to write

$$
\mathrm{E} \tau_{u}=\lim _{\lambda \downarrow 0} \frac{\kappa(\lambda, 0)}{\lambda} V_{H}(u)=\mathrm{E} L_{1}^{-1} V_{H}(u), \quad u>0,
$$

where $V_{H}(u)=\int_{0}^{\infty} \mathrm{P}\left(H_{t} \leq u\right) \mathrm{d} t$ is the renewal function associated with $H$. Now fix $c \in$ $(0, \infty)$.

(a)(i) For $u \rightarrow \infty$, by the elementary renewal theorem (see [29, Corollary 5.3, p. 114]; note that there is no nonlattice restriction on the support of $\Pi_{X}$, and the case $\mathrm{E} H_{1}=\infty$ is covered, e.g. in [23, Theorem 4.1, p. 51]) we have

$$
\lim _{u \rightarrow \infty} \frac{V_{H}(u)}{u}=\frac{1}{\mathrm{E} H_{1}} \in[0, \infty),
$$

so we see that $\lim _{u \rightarrow \infty} \mathrm{E} \tau_{u} / u=1 / c$ for some $c \in(0, \infty)$ if and only if $\mathrm{E} H_{1}<\infty$ and $\mathrm{E} L_{1}^{-1}<\infty$, and then $c=\mathrm{E} H_{1} / \mathrm{E} L_{1}^{-1}$. Since $\mathrm{E} H_{1}<\infty$ is equivalent to $0<\mathrm{E} X_{1} \leq$ $\mathrm{E}\left|X_{1}\right|<\infty$ when $X_{t} \rightarrow \infty$ a.s. by Theorem 8 of [17], we have only to observe that, by Wald's equation for Lévy processes [24], E $H_{1} / \mathrm{E} L_{1}^{-1}=\mathrm{E} X_{1}$.

(a)(ii) For $u \downarrow 0$, assume that $\mathrm{E} L_{1}^{-1}<\infty$ and $d_{H}>0$. Since $\mathscr{H}_{t} \geq d_{H} t, t \geq 0$, it follows easily that

$$
\begin{aligned}
V_{\mathcal{H}}(u) & :=\int_{0}^{\infty} \mathrm{P}\left(\mathscr{H}_{t} \leq u\right) \mathrm{d} t \\
& =\int_{0}^{u / d_{H}} \mathrm{P}\left(\mathscr{H}_{t} \leq u\right) \mathrm{d} t \\
& \leq \mathrm{e}^{q u / d_{H}} \int_{0}^{u / d_{H}} \mathrm{e}^{-q t} \mathrm{P}\left(\mathscr{H}_{t} \leq u\right) \mathrm{d} t \\
& =\mathrm{e}^{q u / d_{H}} V_{H}(u),
\end{aligned}
$$

while, trivially, $V_{H}(u) \leq V_{\mathcal{H}}(u)$. Thus, by Theorem III.5 of [7], which applies to proper subordinators, we have

$$
\lim _{u \downarrow 0} \frac{V_{H}(u)}{u}=\frac{1}{d_{H}},
$$

and so $\lim _{u \downarrow 0} \mathrm{E} \tau_{u} / u=\mathrm{E} L_{1}^{-1} / d_{H}$ by (5.10). Conversely, $\lim _{u \downarrow 0} \mathrm{E} \tau_{u} / u=1 / c$ implies by 
(5.10) that (5.12) holds with $d_{H}$ replaced by $c$ E $L_{1}^{-1}>0$. By Lemma 4 of [14, p. 52] we have

$$
\frac{V_{H}(u)}{u} \asymp \frac{1}{d_{H}+\int_{0}^{u} \bar{\Pi}_{H}(y) \mathrm{d} y+u q} \quad \text { for all } u>0 .
$$

Hence, $d_{H}>0$, since $d_{H}=0$ would imply that $\lim _{u \downarrow 0} V_{H}(u) / u=\infty$, a contradiction.

(b) In the case $c=0$, we see from (5.10) and (5.11) that $\lim _{u \rightarrow \infty} \mathrm{E} \tau_{u} / u$ exists and is in $[0, \infty)$ when $\mathrm{E} \tau_{u}<\infty$ for all $u>0$. When $\lim _{u \rightarrow 0} \mathrm{E} \tau_{u} / u=\infty,(5.10)$ and (5.13) show that $d_{H}=0$, and conversely.

(c) For the case $c=\infty$, supposing that $\mathrm{E} \tau_{u}<\infty$ for each $u>0$, (5.10) and (5.11) show that $\lim _{u \rightarrow \infty} \mathrm{E} \tau_{u} / u=0$ if and only if $\mathrm{E} L_{1}^{-1}<\infty$ and $\mathrm{E} H_{1}=\infty$, while, for $u \downarrow 0$, the case $c=\infty$ cannot arise; $\liminf _{u \rightarrow 0} \mathrm{E} \tau_{u} / u>0$ follows from (5.10) and (5.13).

The following lemma, which is a Lévy process version of a result of [30] for the random walk, is needed in the proofs of Theorems 2.4 and 2.5 .

Lemma 5.2. (Uniform integrability of $\tau_{u}$.) Suppose that $X$ is a Lévy process with $0<\mathrm{E} X_{1} \leq$ $\mathrm{E}\left|X_{1}\right|<\infty$ and $\tau_{u}=\inf \left\{t>0: X_{t}>u\right\}, u>0$. Then $\tau_{u} / u, u>0$, are uniformly integrable as $u \rightarrow \infty$, i.e.

$$
\lim _{x \rightarrow \infty} \limsup _{u \rightarrow \infty} \mathrm{E}\left(\frac{\tau_{u}}{u} \mathbf{1}_{\left\{\tau_{u} / u>x\right\}}\right)=0
$$

Proof. The random walk result of [30] can be transferred using a stochastic bound due to Doney [13]. First consider the case when $\Pi \equiv 0$. Then $X_{t}=t \gamma+\sigma B_{t}$, where $\gamma=\mathrm{E} X_{1}>0$, $\sigma \geq 0$, and $\left(B_{t}\right)_{t \geq 0}$ is a standard Brownian motion. In this case $\tau_{u}$ has an inverse Gaussian distribution and $\mathrm{E} \tau_{u}^{2}=u \sigma^{2} / \gamma^{3}$, which immediately implies the uniform integrability of $\tau_{u} / u$.

So, assume that $\Pi$ is not identically 0 . Then $\bar{\Pi}\left(x_{0}\right)>0$ for some $x_{0}>0$, and by rescaling if necessary we can assume that $c_{1}:=\bar{\Pi}(1)>0$. As in [18], let $\sigma_{0}=0$ and let $\sigma_{n}, n=$ $1,2, \ldots$, be the successive times at which $X$ takes a jump of absolute value greater than 1 . Then the $e_{i}:=\sigma_{i}-\sigma_{i-1}$ are i.i.d. exponential random variables with $\mathrm{E}\left(e_{1}\right)=1 / c_{1}$. Define $S_{n}:=X_{\sigma_{n}}, n=1,2, \ldots$, and $\tau_{u}^{S}=\min \left\{n \geq 1: S_{n}>u\right\}, u>0$. Then $S_{n}$ is a random walk with step distribution $Y_{i}:=X_{\sigma_{i}}-X_{\sigma_{i-1}} \stackrel{\mathrm{D}}{=} X_{e_{1}}$. By Wald's equation, $\mathrm{E} Y_{1}=\mathrm{E} X_{1} / c_{1}>0$. Thus, by [30], the $\tau_{u}^{S} / u$ are uniformly integrable.

Now we can use similar calculations as in [18, p. 287] to bound the expression on the lefthand side of (5.14) in terms of a similar expression involving $\tau_{u}^{S}$. For any $Z \geq 0$ and any $a>0$,

$$
\mathrm{E}(Z ; Z>a)=\int_{z>a} \mathrm{P}(Z>z) \mathrm{d} z+a \mathrm{P}(Z>a) .
$$

Taking $u>0, x>0$, and $c>1 / c_{1}$, we obtain

$$
\mathrm{E}\left(\frac{\tau_{u}}{u c} \mathbf{1}_{\left\{\tau_{u} / u c>x\right\}}\right)=u^{-1} \int_{y>x u} \mathrm{P}\left(\tau_{u}>y c\right) \mathrm{d} y+x \mathrm{P}\left(\tau_{u}>x u c\right) .
$$

By Theorem 2.1 we have $\tau_{u} / u \stackrel{\mathrm{P}}{\rightarrow} 1 / \mathrm{E} X_{1}$ as $u \rightarrow \infty$. So the second term on the right-hand side of (5.16) tends to 0 as $u \rightarrow \infty$ once $x c>1 / \mathrm{E} X_{1}$. As in [18, p. 287], we have

$$
\mathrm{P}\left(\sigma_{j} \leq \tau_{u}<\sigma_{j+1}\right)=\mathrm{P}\left(\tilde{m}_{0} \leq u, \tau_{u-\tilde{m}_{0}}^{S}=j\right), \quad j \geq 1,
$$

where $\tilde{m}_{0}$ is a finite random variable independent of $\left(S_{n}\right)_{n=1,2, \ldots}$. The first term on the right-hand 
side of (5.16) is bounded by

$$
u^{-1} \sum_{n \geq\lfloor x u\rfloor} \mathrm{P}\left(\tau_{u}>n c\right)
$$

and now we argue as follows:

$$
\begin{gathered}
u^{-1} \sum_{n \geq\lfloor x u\rfloor} \mathrm{P}\left(\tau_{u}>n c\right) \leq u^{-1} \sum_{n \geq\lfloor x u\rfloor} \mathrm{P}\left(\tau_{u} \geq \sigma_{n}\right)+u^{-1} \sum_{n \geq\lfloor x u\rfloor} \mathrm{P}\left(\sigma_{n}>n c\right) \\
\leq u^{-1} \sum_{j \geq\lfloor x u\rfloor}(j-\lfloor x u\rfloor+1) \mathrm{P}\left(\sigma_{j} \leq \tau_{u}<\sigma_{j+1}\right) \\
+u^{-1} \sum_{n \geq 1} \mathrm{P}\left(\sum_{i=1}^{n} e_{i}>n c\right) .
\end{gathered}
$$

Since the $e_{i}$ are i.i.d. with a finite exponential moment and $\mathrm{E} e_{i}=1 / c_{1}<c$, the sum in the second term on the right-hand side of (5.17) is convergent, and, hence, this term is $o(1)$ as $u \rightarrow \infty$. The first term on the right-hand side of (5.17) is

$$
\begin{aligned}
u^{-1} & \sum_{j \geq\lfloor x u\rfloor}(j-\lfloor x u\rfloor+1) \mathrm{P}\left(\tilde{m}_{0} \leq u, \tau_{u-\tilde{m}_{0}}^{S}=j\right) \\
& =u^{-1} \sum_{j \geq\lfloor x u\rfloor}(j-\lfloor x u\rfloor+1) \int_{0}^{u} \mathrm{P}\left(\tau_{u-y}^{S}=j\right) \mathrm{P}\left(\tilde{m}_{0} \in \mathrm{d} y\right) \\
& =u^{-1} \sum_{n \geq\lfloor x u\rfloor} \int_{0}^{u} \mathrm{P}\left(\tau_{u-y}^{S} \geq n\right) \mathrm{P}\left(\tilde{m}_{0} \in \mathrm{d} y\right) \\
& \leq u^{-1} \sum_{n \geq\lfloor x u\rfloor} \mathrm{P}\left(\tau_{u}^{S} \geq n\right) \\
& \leq u^{-1} \int_{y>\lfloor x u\rfloor-1} \mathrm{P}\left(\tau_{u}^{S}>y\right) \mathrm{d} y \\
& \leq \mathrm{E}\left(\frac{\tau_{u}^{S}}{u} \mathbf{1}_{\left\{\tau_{u}^{S} / u>x / 2\right\}}\right)
\end{aligned}
$$

if $x u \geq 4$, where the last inequality comes from (5.15). Since the $\tau_{u}^{S} / u$ are uniformly integrable by Lai's result, we get (5.14).

Remark 5.1. Lemma 5.2 could be used to give an alternative proof of Theorem 2.3(a)(i) from Theorem 2.2. This approach however would not work for Theorem 2.3(a)(ii), since the $\tau_{u} / u$ are not uniformly integrable as $u \downarrow 0$. This is because the almost-sure limit in Theorem 2.2 does not agree with the limit in mean in Theorem 2.3 as $u \downarrow 0$.

Proof of Theorem 2.4. (a) We first observe that (2.12) is equivalent to

$$
\lim _{x \rightarrow 1 / L} \frac{\kappa(0, x)-\kappa(0,0)}{\kappa(\xi x, x)}=\frac{c}{c+\xi} \quad \text { for each } \xi>0 .
$$

When $L=\infty,(5.18)$ holds because $\kappa(0,0)=q=0$ by our assumption that $\lim _{\sup } \operatorname{su}_{t \rightarrow \infty} X_{t}=$ $\infty$. When $L=0$, we have, by (1.1), $\kappa(\xi x, x) \rightarrow \infty$ as $x \rightarrow 1 / L$ for each $\xi>0$ unless $d_{H}=d_{L^{-1}}=0$ and $\Pi_{L^{-1}, H}$ is a finite measure. But this is impossible since 0 is regular for $(0, \infty)$, so $(5.18)$ is equivalent when $L=0$ also. 
From (1.2) we have, for $u>0$ and $v>0$,

$$
\mu \int_{u \geq 0} \mathrm{e}^{-\mu u} \mathrm{E}\left(\mathrm{e}^{-\nu G_{\tau_{u}}} ; \tau_{u}<\infty\right) \mathrm{d} u=\frac{\kappa(0, \mu)-\kappa(0,0)}{\kappa(\nu, \mu)} .
$$

Take $y>0$, and replace $\mu$ by $\mu / y, u$ by $u y$, and $\nu$ by $v / y$ in this equation to get

$$
\mu \int_{u \geq 0} \mathrm{e}^{-\mu u} \mathrm{E}\left(\mathrm{e}^{-\nu G_{\tau_{u y-}} / y} ; \tau_{u y}<\infty\right) \mathrm{d} u=\frac{\kappa(0, \mu / y)-\kappa(0,0)}{\kappa(\nu / y, \mu / y)} .
$$

Equation (2.11) implies that $G_{\tau_{u y-}} / y \stackrel{\mathrm{P}}{\rightarrow} u / c$ as $y \rightarrow L$ for each $u>0$. So the left-hand side of (5.19) tends to $c /(c+v / \mu)$ as $y \rightarrow L$, and then (2.12) follows from (5.18) and the right-hand side of (5.19). The proof that (2.12) implies (2.11) is analogous to that in Theorem 2.1.

(b) By the strong law when $L=\infty$ and by Theorem 39 of [14] when $L=0$, it suffices to prove that, for $c \in[0, \infty)$,

$$
\lim _{t \rightarrow L} \frac{X_{t}}{t}=c \quad \text { a.s., }
$$

if and only if

$$
\lim _{u \rightarrow L} \frac{G_{\tau_{u}-}}{u}=\frac{1}{c} \quad \text { a.s. }
$$

A simple pathwise argument shows that (5.20) implies (5.21) when $c>0$, but the case $c=0$ is a little trickier. Since the following argument works whenever $c \in[0, \infty)$, we prove it under that assumption. So assume that (5.20) holds with $c \in[0, \infty)$. Then $\bar{X}_{t} / t \rightarrow c$ a.s. as $t \rightarrow L$. If (5.21) fails then

$$
\liminf _{u \rightarrow L} \frac{G_{\tau_{u}-}}{u}<\frac{1}{c} \text { a.s., }
$$

since under (5.20), $G_{\tau_{u}-} / u \leq \tau_{u} / u \rightarrow 1 / c$ a.s. as $u \rightarrow L$, by Theorem 2.2. Now consider the random level $Z_{u}=\bar{X}_{\tau_{u}-}$, and observe that

$$
\tau_{Z_{u}}=\tau_{u}, \quad \bar{X}_{G_{\tau_{u}-}}=\bar{X}_{\tau_{u}-}=Z_{u} .
$$

Writing

$$
\frac{\bar{X}_{G_{\tau_{u}-}}}{G_{\tau_{u}-}}=\frac{Z_{u}}{u} \frac{u}{G_{\tau_{u}-}}
$$

it then follows that

$$
\liminf _{u \rightarrow L} \frac{Z_{u}}{u}<1 \quad \text { a.s. }
$$

Thus,

$$
\limsup _{u \rightarrow L} \frac{X_{\tau_{Z_{u}}}}{Z_{u}}=\limsup _{u \rightarrow L} \frac{X_{\tau_{u}}}{Z_{u}} \geq \limsup _{u \rightarrow L} \frac{u}{Z_{u}}>1 \quad \text { a.s. }
$$

In particular, it is not the case that $\lim _{v \rightarrow L} X_{\tau_{v}} / v=1$ a.s. This implies that $\mathrm{E}|X|=\infty$ when $L=\infty$, by Theorem 8 of [17], and $X$ is not of bounded variation when $L=0$, by Theorem 4 of [17]. In either case (5.20) fails to hold, which is a contradiction.

Conversely, assume that (5.21) holds for some $c \in[0, \infty)$. Then, for any $a>c, G_{\tau_{u}-}>$ $u a^{-1}$ eventually. Hence, $\bar{X}_{u a^{-1}} \leq u$ eventually. This implies that

$$
\limsup _{t \rightarrow L} \frac{X_{t}}{t} \leq c \quad \text { a.s. }
$$


If $L=\infty$ then arguing as in the proof of Theorem 2.2(a)(i), (5.22) implies that $0 \leq \mathrm{E} X_{1} \leq$ $\mathrm{E}\left|X_{1}\right|<\infty$ and so (5.20) holds with $c=\mathrm{E} X_{1} \geq 0$. Since (5.20) implies (5.21), the constant $c$ for which (5.21) was assumed to hold must also have been $c=\mathrm{E} X_{1}$, completing the proof of (i). If $L=0$ then (5.22) forces $X$ to have bounded variation with $d_{X} \geq 0$ since 0 is regular for $(0, \infty)$. In that case (5.20) holds with $c=d_{X}$, and so again since (5.20) implies (5.21), the constant $c$ for which (5.21) was assumed to hold must also have been $c=d_{X}$, completing the proof of (ii).

(c) Finally, suppose that $0<\mathrm{E} X_{1} \leq \mathrm{E}\left|X_{1}\right|<\infty$. Then (5.21) holds with $c=\mathrm{E} X_{1}$ and, further, the $G_{\tau_{u}-} / u$ are uniformly integrable as $u \rightarrow \infty$ by Lemma 5.2. Thus, we get $\lim _{u \rightarrow \infty} \mathrm{E}\left(G_{\tau_{u}-} / u\right)=1 / \mathrm{E} X_{1}$.

Proof of Theorem 2.5. Since $0<\mathrm{E} X_{1} \leq \mathrm{E}\left|X_{1}\right|<\infty$, we have, by Theorems 2.2 and 2.4,

$$
\lim _{u \rightarrow \infty} \frac{G_{\tau_{u}-}}{u}=\lim _{u \rightarrow \infty} \frac{\tau_{u}}{u}=\frac{1}{\mathrm{E} X_{1}} \text { a.s. }
$$

The assumptions on $X$ imply that $H$ does not have a lattice jump distribution, and, hence, it follows from [10] that

$$
X_{\tau_{u}}-u \stackrel{\mathrm{D}}{\rightarrow} Y \quad \text { as } u \rightarrow \infty,
$$

where $Y$ is the random variable defined in the statement of Theorem 2.5. Since $\tau_{u} / u$, and, consequently, $G_{\tau_{u}-} / u$ also, are uniformly integrable as $u \rightarrow \infty$, by Lemma 5.2 , the result follows.

\section{Proofs for Section 3}

We assume throughout this section the setup of Section 3. Let $\mathcal{F}_{\tau_{u}}$ be the $\sigma$-algebra generated by $X$ up to time $\tau_{u}$. By Corollary 3.11 of [29], for any $Z_{u}$ which is nonnegative and measurable with respect to $\mathcal{F}_{\tau_{u}}$, we have

$$
\mathrm{E}\left(Z_{u} ; \tau_{u}<\infty\right)=\mathrm{E}^{*}\left(Z_{u} \mathrm{e}^{-\nu_{0} X_{\tau_{u}}}\right) .
$$

This immediately yields the following lemma, which can be found in Theorem IV.7.1 of [3] for compound Poisson processes with negative drift. Our proof is analogous to that in [3].

Lemma 6.1. Suppose that $\mu^{*}<\infty$ and that, for $u>0, Y_{u}$ are $\mathcal{F}_{\tau_{u}}$-measurable random variables such that $Y_{u} \stackrel{\mathrm{p}^{*}}{\rightarrow} 0$ as $u \rightarrow \infty$. Then $Y_{u} \stackrel{\mathrm{p}^{(u)}}{\longrightarrow} 0$.

Proof. For $\varepsilon>0$, by (6.1),

$$
\mathrm{P}^{(u)}\left(\left|Y_{u}\right|>\varepsilon\right)=\frac{\mathrm{P}\left(\left|Y_{u}\right|>\varepsilon, \tau_{u}<\infty\right)}{\mathrm{P}\left(\tau_{u}<\infty\right)}=\frac{\mathrm{E}^{*}\left(\mathrm{e}^{-\nu_{0}\left(X_{\tau_{u}}-u\right)} ;\left|Y_{u}\right|>\varepsilon\right)}{\mathrm{e}^{\nu_{0} u} \mathrm{P}\left(\tau_{u}<\infty\right)} .
$$

Since $\mu^{*}<\infty$, by (3.3) the denominator here is bounded away from 0 , hence the result.

Proof of Theorem 3.2. Since $X$ is a Lévy process under $\mathrm{P}^{*}$ with $\mathrm{E}^{*} X_{1}=\mu^{*} \in(0, \infty)$, it follows easily from the strong law that

$$
\frac{X_{\tau_{u}}}{u} \rightarrow 1, \quad \frac{G_{\tau_{u}-}}{u} \rightarrow \frac{1}{\mu^{*}}, \quad \text { and } \quad \frac{\tau_{u}}{u} \rightarrow \frac{1}{\mu^{*}}, \quad \mathrm{P}^{*} \text {-a.s. as } u \rightarrow \infty .
$$

Equation (3.4) is then immediate from Lemma 6.1. 
For (3.5), use Theorem 2.5, (3.3), and (6.1) to deduce that, as $u \rightarrow \infty$,

$$
\frac{\mathrm{E}^{(u)} \tau_{u}}{u}=\frac{\mathrm{E}\left(\tau_{u} ; \tau_{u}<\infty\right)}{u \mathrm{P}\left(\tau_{u}<\infty\right)}=\frac{\mathrm{E}^{*}\left(\tau_{u} \mathrm{e}^{-\nu_{0}\left(X_{\tau_{u}}-u\right)}\right)}{u \mathrm{e}^{v_{0} u} \mathrm{P}\left(\tau_{u}<\infty\right)} \rightarrow \frac{1}{C \mu^{*}} \mathrm{E}^{*} \mathrm{e}^{-v_{0} Y}=\frac{1}{\mu^{*}} .
$$

The limit involving $G_{\tau_{u}}$ is similar. For the final limit in (3.5), first observe that

$$
\frac{\mathrm{E}^{(u)} X_{\tau_{u}}}{u}=\frac{\mathrm{E}\left(X_{\tau_{u}} ; \tau_{u}<\infty\right)}{u \mathrm{P}\left(\tau_{u}<\infty\right)} \sim \frac{\mathrm{E}^{*}\left(X_{\tau_{u}} \mathrm{e}^{-\nu_{0}\left(X_{\tau_{u}}-u\right)}\right)}{C u} .
$$

Now let $O_{u}:=X_{\tau_{u}}-u, u>0$. Then $\left(X_{\tau_{u}} / u\right) \mathrm{e}^{-v_{0} O_{u}}$ is uniformly integrable because, for $x>1$ and $c_{0}:=\sup _{y \geq 0}\left(y \mathrm{e}^{-v_{0} y}\right)=\left(\mathrm{e}_{0}\right)^{-1}$, we have

$$
\begin{aligned}
\mathrm{E}^{*}\left(\frac{X_{\tau_{u}}}{u} \mathrm{e}^{-v_{0} O_{u}} \mathbf{1}_{\left\{X_{\tau_{u}} / u>x\right\}}\right) & =\mathrm{E}^{*}\left(\frac{O_{u}}{u} \mathrm{e}^{-v_{0} O_{u}} \mathbf{1}_{\left\{O_{u}>(x-1) u\right\}}\right)+\mathrm{E}^{*}\left(\mathrm{e}^{-v_{0} O_{u}} \mathbf{1}_{\left\{O_{u}>(x-1) u\right\}}\right) \\
& \leq \frac{c_{0}}{u}+\mathrm{e}^{-v_{0}(x-1) u} .
\end{aligned}
$$

Letting $u \rightarrow \infty$ then $x \rightarrow \infty$ shows the uniform integrability. Since $X_{\tau_{u}}-u \stackrel{\mathrm{D}^{*}}{\rightarrow} Y$ and $X_{\tau_{u}} / u \stackrel{\mathrm{P}^{*}}{\rightarrow} 1$ by (6.2), we have

$$
\frac{\mathrm{E}^{(u)} X_{\tau_{u}}}{u} \sim C^{-1} \mathrm{E}^{*}\left(\frac{X_{\tau_{u}}}{u} \mathrm{e}^{-v_{0} O_{u}}\right) \rightarrow C^{-1} \mathrm{E}^{*} \mathrm{e}^{-v_{0} Y}=1,
$$

completing the proof.

\section{Appendix A}

Proof of Lemma 5.1. We first construct a Lévy process satisfying (5.5). For the characteristics of $X$, we take $\gamma=-2, \sigma=0$, and the Lévy measure given by

$$
\begin{gathered}
\bar{\Pi}_{X}^{+}(x)=\frac{1}{x|\ln x|}, \quad \bar{\Pi}_{X}^{-}(x)=\bar{\Pi}_{X}^{+}(x)+\frac{\ln 2}{x(\ln x)^{2}}, \quad 0<x<\frac{1}{2} \\
\bar{\Pi}_{X}^{+}(x)=\bar{\Pi}_{X}^{-}(x)=0, \quad x \geq \frac{1}{2} .
\end{gathered}
$$

Then $X$ is not of bounded variation since

$$
\int(|x| \wedge 1) \Pi(\mathrm{d} x)=\infty
$$

and, consequently, 0 is regular for $(0, \infty)$. Furthermore, one can easily check that (2.7) holds with $c=-1$, and so

$$
\frac{X_{t}}{t} \stackrel{\mathrm{P}}{\rightarrow}-1 \quad \text { as } t \downarrow 0
$$

by Theorem 2.1 of [16]. Also, the argument given in Theorem 2.1 that (2.5) implies (2.4) when $L=0$ shows that, under (2.7),

$$
\mathrm{P}\left(\sup _{0 \leq s \leq t}\left|X_{s}+s\right|>\varepsilon t\right) \rightarrow 0 \quad \text { as } t \downarrow 0 .
$$

From this we conclude that

$$
\frac{\bar{X}_{t}}{t} \stackrel{\mathrm{P}}{\rightarrow} 0 \quad \text { as } t \downarrow 0
$$

completing the example. 
We now construct a Lévy process satisfying (5.6). This is based on Example 3.5 of [27], which constructs a random walk $S_{n}=\sum_{i=1}^{n} Y_{i}$ with i.i.d. summands $Y_{i}$ which satisfy

$$
\frac{S_{n}}{n} \stackrel{\mathrm{P}}{\rightarrow}-\infty \quad \text { and } \quad \frac{\bar{S}_{n}}{n}=\frac{\max _{1 \leq j \leq n} S_{j}}{n} \stackrel{\mathrm{P}}{\rightarrow} \infty .
$$

This is done by finding a random walk which is negatively relatively stable (NRS) as $n \rightarrow \infty$, i.e. with

$$
\frac{S_{n}}{D(n)} \stackrel{\mathrm{P}}{\rightarrow}-1 \quad(n \rightarrow \infty)
$$

for a norming sequence $D(n)>0$ with $D(n) / n \rightarrow \infty$ satisfying

$$
\lim _{n \rightarrow \infty} \sum_{j=\ell(n)}^{n} \mathrm{P}\left(S_{1}>x D(j)\right)=\infty
$$

where $\ell(n)$ is the inverse to $D(n)$. The sequences $D(n)$ and $\ell(n)$ are strictly increasing to $\infty$ as $n \rightarrow \infty$ and satisfy $D(n)=-n A(D(n))$ (where $A(\cdot)$ is defined in $(2.1)$ ) and $\ell(n)=-n / A(n)$. The function $-A(x)$ is positive for large enough $x$, slowly varying as $x \rightarrow \infty$, and tends to $\infty$ as $x \rightarrow \infty$.

(i) Consider first the case $L=\infty$. Let $\left(N_{t}\right)_{t \geq 0}$ be a Poisson process of rate 1 independent of the $Y_{i}$, and set $X_{t}:=S_{N_{t}}, t \geq 0$, where $S_{n}$ is as in (A.1). Then the compound Poisson process $X_{t}$ satisfies (5.6) with $L=\infty$. This is fairly straightforward to check and we omit the details.

(ii) Now consider the case $L=0$. For this, we have to modify Example 3.5 of [27] to work as $t \downarrow 0$. Details are as follows.

We construct a Lévy process $X_{t}$ which is NRS as $t \downarrow 0$, i.e. is such that

$$
\frac{X_{t}}{b(t)} \stackrel{\mathrm{P}}{\rightarrow}-1 \quad \text { as } t \downarrow 0
$$

for a nonstochastic function $b(t)>0$, with $b(t) \downarrow 0$ and $b(t) / t \rightarrow \infty$ as $t \downarrow 0$. To do this, it will be useful to summarize here some properties concerning (negative) relative stability at 0 of $X_{t}$; for reference, see [16] (and replace $X$ by $-X$ ). We assume that $\bar{\Pi}_{X}(x)>0$ for all $x>0$. We then have $X_{t} \in$ NRS if and only if $\sigma=0$ and the function $A(x)$ defined in (2.1) is strictly negative for all small enough $x, x \leq x_{0}$, say, and satisfies

$$
\lim _{x \downarrow 0} \frac{A(x)}{x \bar{\Pi}_{X}(x)}=-\infty .
$$

When $A(x)<0$ for $x \leq x_{0}$, there is an $x_{1} \leq x_{0}$ so that the function

$$
D(x):=\sup \left\{y \geq x_{0}: \frac{-A(y)}{y} \geq \frac{1}{x}\right\}, \quad 0<x \leq x_{1},
$$

is strictly positive and finite, and satisfies

$$
D(x)=-x A(D(x))
$$

for all $x \leq x_{1}$. It is easily seen to be strictly increasing on $x \leq x_{1}$ (by the continuity of $y \mapsto-A(y) / y$ ) with $D(0)=0$. Thus, for small enough $y$, we can define the inverse function

$$
\ell(y)=\sup \{x: D(x) \leq y\}=\inf \{x: D(x)>y\} .
$$


When (A.3) holds, $-A(x)$ is slowly varying as $x \downarrow 0$, and, as a consequence, $D(x)$ and $\ell(x)$ are both regularly varying with index 1 as $x \downarrow 0$ (see [11, Theorem 1.5.12]). Also, when (A.3) holds, it is easy to check that $-A(y) / y$ is strictly decreasing for small enough $y$, so $\ell(y)$ is continuous and strictly increasing, with $\ell(0)=0$, and

$$
\ell(y)=\frac{y}{-A(y)}
$$

for small enough $y$. Finally, we can take $b(t)=D(t)$ in (A.2) to get negative relative stability of $X$ in the form:

$$
\frac{X_{t}}{D(t)} \stackrel{\mathrm{P}}{\rightarrow}-1 \quad \text { as } t \downarrow 0 .
$$

To construct the process required in the lemma, we will specify a Lévy measure $\Pi_{X}$ for $X$ such that

$$
\frac{D(t)}{t} \rightarrow \infty \quad \text { as } t \downarrow 0 ;
$$

consequently, $\ell(t) / t \rightarrow 0$, and

$$
\int_{\ell(t)}^{t} \bar{\Pi}_{X}^{+}(x D(s)) \mathrm{d} s \rightarrow \infty \quad \text { as } t \downarrow 0
$$

for all $x>0$. Equation (A.5) then implies that $X_{t} / t \stackrel{\mathrm{P}}{\rightarrow}-\infty$ as $t \downarrow 0$. We claim that in addition (A.5)-(A.7) imply that

$$
\frac{\bar{X}_{t}}{t} \stackrel{\mathrm{P}}{\rightarrow} \infty \quad \text { as } t \downarrow 0 .
$$

To prove (A.8) from (A.5)-(A.7), fix $t>0$ and $x>1$, and write

$$
\begin{aligned}
& \mathrm{P}\left(\bar{X}_{t}>x t\right)=\lim _{n \rightarrow \infty} \mathrm{P}\left(\max _{1 \leq j \leq n t} X(j / n)>x D(\ell(t))\right) \\
& \geq \liminf _{n \rightarrow \infty} \sum_{n \ell(t) \leq j \leq n t} \mathrm{P}(X((j-1) / n)>-x D(j / n), \\
&\left.\max _{j+1 \leq k \leq n t} \frac{\Delta(k / n)}{D(k / n)} \leq 2 x<\frac{\Delta(j / n)}{D(j / n)}\right),
\end{aligned}
$$

where

$$
\Delta(k / n):=X(k / n)-X((k-1) / n), \quad k=1,2, \ldots,
$$

are i.i.d. with distribution the same as that of $X(1 / n)$. Given $\varepsilon \in(0,1)$, by (A.5), there is a $t_{0}>0$ such that $\mathrm{P}(X((j-1) / n)>-x D(j / n))>1-\varepsilon$ when $j / n \leq t \leq t_{0}$. Thus, keeping $t \leq t_{0}$

$$
\mathrm{P}\left(\bar{X}_{t}>x t\right) \geq(1-\varepsilon) \liminf _{n \rightarrow \infty} \sum_{n \ell(t) \leq j \leq n t} \mathrm{P}\left(\max _{j+1 \leq k \leq n t} \frac{\Delta(k / n)}{D(k / n)} \leq 2 x<\frac{\Delta(j / n)}{D(j / n)}\right) .
$$

Here the sum equals

$$
\begin{aligned}
& \mathrm{P}(\Delta(j / n)>2 x D(j / n) \text { for some } j \in[n \ell(t), n t]) \\
& \quad=1-\prod_{n \ell(t) \leq j \leq n t} \mathrm{P}(X(1 / n) \leq 2 x D(j / n)) \\
& \quad \geq 1-\exp \left(-\sum_{n \ell(t) \leq j \leq n t} \mathrm{P}(X(1 / n)>2 x D(j / n))\right) .
\end{aligned}
$$


Noting that

$$
\begin{aligned}
n \int_{\ell(t)}^{t+1 / n} \mathrm{P}(X(1 / n)>2 x D(s)) \mathrm{d} s & \leq n \sum_{n \ell(t) \leq j \leq n t} \int_{j / n}^{(j+1) / n} \mathrm{P}(X(1 / n)>2 x D(s)) \mathrm{d} s \\
& \leq \sum_{n \ell(t) \leq j \leq n t} \mathrm{P}(X(1 / n)>2 x D(j / n)) \mathrm{d} s,
\end{aligned}
$$

and employing the fact that $\lim _{n \rightarrow \infty} n \mathrm{P}(X(1 / n)>a)=\bar{\Pi}_{X}^{+}(a)$ for all $a>0$ (cf. [7, p. 39]), we get

$$
\begin{aligned}
\mathrm{P}\left(\bar{X}_{t}>x t\right) & \geq(1-\varepsilon) \liminf _{n \rightarrow \infty}\left(1-\exp \left(-n \int_{\ell(t)}^{t+1 / n} \mathrm{P}(X(1 / n)>2 x D(s)) \mathrm{d} s\right)\right) \\
& =(1-\varepsilon)\left(1-\exp \left(-\int_{\ell(t)}^{t} \bar{\Pi}_{X}^{+}(2 x D(s)) \mathrm{d} s\right)\right) .
\end{aligned}
$$

The last expression tends to 1 as $t \downarrow 0$ then $\varepsilon \downarrow 0$, provided that (A.7) holds. Thus, (A.8) will follow from (A.5)-(A.7), as claimed.

It remains to give an example where (A.5)-(A.7) hold. Define

$$
L(x)=\mathrm{e}^{(-\log x)^{\beta}}, \quad 0<x<\mathrm{e}^{-1},
$$

and keep $\frac{1}{2}<\beta<1$. Choose a Lévy measure $\Pi_{X}$ which satisfies

$$
\bar{\Pi}_{X}^{+}(x)=-2 L^{\prime}(x)=\frac{2 \beta(-\log x)^{\beta-1} L(x)}{x}
$$

and

$$
\bar{\Pi}_{X}^{-}(x)=-L^{\prime}(x)=\frac{1}{2} \bar{\Pi}_{X}^{+}(x)
$$

for small enough $x, x \leq x_{0}$, say. (Note that $\bar{\Pi}_{X}^{+}(x)$ and $\bar{\Pi}_{X}^{-}(x)$ are infinite at 0 and decrease to 0 as $x \rightarrow \infty$.) A straightforward calculation using (2.1) gives, for $x>0$,

$$
A(x)=\gamma+\bar{\Pi}_{X}^{+}(1)-\bar{\Pi}_{X}^{-}(1)+1-L(x) .
$$

Thus, $A(x) \rightarrow-\infty$ as $x \downarrow 0$, and

$$
\frac{-A(x)}{x \bar{\Pi}_{X}(x)} \sim \frac{L(x)}{-3 L^{\prime}(x)} \sim \frac{(-\log x)^{1-\beta}}{3 \beta} \rightarrow \infty \quad \text { as } x \downarrow 0 .
$$

Thus, by (A.3), $X$ is NRS at 0 and (A.5) holds with $D(t)$ as in (A.4). We have $D(t)=-t A(D(t))$ and $\ell(t)=-t / A(t)$ for small enough $t>0$. Since $-A(x) \rightarrow \infty$ as $x \downarrow 0$, (A.6) also holds. Now $L(x)$ is slowly varying as $x \downarrow 0$, so $\bar{\Pi}_{X}^{+}(x)$ is regularly varying with index -1 as $x \downarrow 0$. It therefore suffices to check (A.7) with $x=1$. Note also that $\log (D(t)) \sim \log t$ as $t \downarrow 0$, 
because $A(\cdot)$ is slowly varying at 0 . Hence, for some constant $c_{2}>0$, as $t \downarrow 0$,

$$
\begin{aligned}
\int_{\ell(t)}^{t} \bar{\Pi}_{X}^{+}(D(s)) \mathrm{d} s & =\int_{\ell(t)}^{t} \frac{D(s) \bar{\Pi}_{X}^{+}(D(s))}{-s A(D(s))} \mathrm{d} s \\
& \sim 3 \beta \int_{\ell(t)}^{t} \frac{(-\log D(s))^{\beta-1}}{s} \mathrm{~d} s \\
& \geq 3 \beta(-\log D(t))^{\beta-1}(\log t-\log \ell(t)) \\
& \geq c_{2}(-\log t)^{2 \beta-1} \\
& \rightarrow \infty
\end{aligned}
$$

(because $\beta>\frac{1}{2}$ ). Thus, (A.7) holds too.

\section{References}

[1] Asmussen, S. (1982). Conditioned limit theorems relating a random walk to its associate, with applications to risk reserve processes and the GI/G/1 queue. Adv. Appl. Prob. 14, 143-170.

[2] Asmussen, S. (1984). Approximations for the probability of ruin within finite time. Scand. Actuarial J. 1984, 31-57. (Correction: 1985(1985), 64.)

[3] Asmussen, S. (2000). Ruin Probabilities. World Scientific, River Edge, NJ.

[4] Asmussen, S. (2003). Applied Probability and Queues, 2nd edn. Springer, New York.

[5] Asmussen, S. And KlüPPElberg, C. (1996). Large deviations results for subexponential tails, with applications to insurance risk. Stoch. Process. Appl. 64, 103-125.

[6] Avram, F. And Usabel, M. (2003). Finite time ruin probabilities with one Laplace inversion. Insurance Math. Econom. 32, 371-377.

[7] Bertoin, J. (1996). Lévy Processes. Cambridge University Press.

[8] Bertoin, J. (1997). Regularity of the half-line for Lévy processes. Bull. Sci. Math. 121, 345-354.

[9] Bertoin, J. and Doney, R. A. (1994). Cramér's estimate for Lévy processes. Statist. Prob. Lett. 21, 363-365.

[10] Bertoin, J., van Harn, K. And Steutel, F. W. (1999). Renewal theory and level passage by subordinators. Statist. Prob. Lett. 45, 65-69.

[11] Bingham, N. H., Goldie, C. M. and Teugels, J. L. (1987). Regular Variation. Cambridge University Press.

[12] Braverman, M. (1997). Suprema and sojourn times of Lévy processes with exponential tails. Stoch. Proc. Appl. 68, 265-283.

[13] Doney, R. A. (2004). Stochastic bounds for Lévy Processes. Ann. Prob. 32, 1545-1552.

[14] Doney, R. A. (2005). Fluctuation Theory for Lévy Processes (Lecture Notes Math. 1897). Springer, Berlin.

[15] Doney, R. A. and Kyprianou, A. E. (2006). Overshoots and undershoots of Lévy processes. Ann. Appl. Prob. 16, 91-106.

[16] Doney, R. A. And Maller, R. A. (2002). Stability and attraction to normality for Lévy processes at zero and infinity. J. Theoret. Prob. 15, 751-792.

[17] Doney, R. A. And Maller, R. A. (2002). Stability of the overshoot for Lévy processes. Ann. Prob. 30, 188-212.

[18] Doney, R. A. And Maller, R. A. (2004). Moments of passage times for Lévy processes. Ann. Inst. H. Poincaré Prob. Statist. 40, 279-297.

[19] Foss, S. And Zachary, S. (2003). The maximum on a random time interval of a random walk with long-tailed increments and negative drift. Ann. Appl. Prob. 13, 37-53.

[20] Grandell, J. (1991). Aspects of Risk Theory. Springer, New York.

[21] Griffin, P. S. AND Maller, R. A. (2011). Path decomposition of ruinous behaviour for a general Lévy insurance risk process. To appear in Ann. Appl. Prob.

[22] Griffin, P. S. And Maller, R. A. (2011). The time at which a Lévy process creeps. Submitted.

[23] Gut, A. (2009). Stopped Random Walks, 2nd edn. Springer, New York.

[24] Hall, W. J. (1970). On Wald's equations in continuous time. J. Appl. Prob. 7, 59-68.

[25] Hao, X. AND TANG, Q. (2009). Asymptotic ruin probabilities of the Lévy insurance model under periodic taxation. ASTIN Bull. 39, 479-494.

[26] Heyde, C. C. And WANG, D. (2009). Finite-time ruin probability with an exponential Lévy process investment return and heavy-tailed claims. Adv. Appl. Prob., 41, 206-224.

[27] Kesten, H. and Maller, R. (1999). Stability and other limit laws for exit times of random walks from a strip or a halfplane. Ann. Inst. H. Poincaré Prob. Statist. 35, 685-734. 
[28] Klüppelberg, C., Kyprianou A. E. and Maller, R. A. (2004). Ruin probabilities and overshoots for general Lévy insurance risk processes. Ann. Appl. Prob. 14, 1766-1801.

[29] Kyprianou, A. E. (2006). Introductory Lectures on Fluctuations of Lévy Processes with Applications. Springer, Berlin.

[30] LaI, T. L. (1975). On uniform integrability in renewal theory. Bull. Inst. Math. Acad. Sinica 3, 99-105.

[31] Park, H. S. And Maller, R. A. (2008). Moment and MGF convergence of overshoots and undershoots for Lévy insurance risk processes. Adv. Appl. Prob. 40, 716-733.

[32] Percheskit, E. A. And Rogozin, B. A. (1969). On joint distributions of random variables associated with fluctuations of a process with independent increments, Theoret. Prob. Appl. 14, 410-423.

[33] Sato, K.-I. (1999). Lévy Processes and Infinitely Divisible Distributions. Cambridge University Press. 